\title{
Surface-Anchored Metal-Organic Frameworks - SURMOFs: A New Material Platform for Sensors
}

\author{
Himanshu Gulati, Peter Lindemann, Lars Heinke, Jianxi Liu, Manuel Tsotsalas, Christof Wöll, $\underline{\text { Hartmut }}$ \\ Gliemann \\ Institute of Functional Interfaces (IFG), Karlsruhe Institute of Technology (KIT), Karlsruhe, Germany \\ Contact: hartmut.gliemann@kit.edu
}

\begin{abstract}
The application of surface-anchored metal-organic frameworks (SURMOFs) as highly porous host materials for molecules in the field of nanoplasmonic sensing (NPS) is reported. SURMOFs are grown by a layer-bylayer procedure on the surface of the NPS sensors, which consist of a quartz glass with $\mathrm{SiO}_{2}$ coated gold nanodisks. As the spectral position of the gold plasmon band depends on the refractive index of the surrounding medium, the loading of the SURMOF pores with guest molecules results in a change of the effective refractive index of the SURMOF and therefore in a spectral shift of the plasmonic band. The combination of SURMOFs that change their optical properties with loading with the method of nanoplasmonic sensing opens new fields of application in sensor technology. Here we show the proof of principle with HKUST-1 as the SURMOF material and with cyclohexane and ethanol as guest molecules.
\end{abstract}

\section{Key words}

Metal-organic frameworks, SURMOF, nanoplasmonic sensing, sensor, refractive index

\section{Introduction}

Metal organic frameworks (MOFs) are highly crystalline and highly porous (up to $6.000 \mathrm{~m}^{2} / \mathrm{g}$ ) molecular solids and consist of two different types of building blocks: (1) metal or metal-oxo units and (2) organic linker molecules acting as struts. Usually, bulk MOFs are prepared by mixing the solutions of both components resulting in the precipitation of a MOF powder by a selfassembling process. Besides the usual application of powder MOFs as highly efficient gas storage material, many other applications came up where the deposition of MOFs on substrates becomes necessary. Several different techniques were developed to immobilize powder MOFs on substrates [1, 2, 3, 4, 5]. However, for applications where the diffusion of molecules into these layers plays a decisive role - such as in sensor technology - these granular and often heterogeneous coatings exhibit a number of major drawbacks: Due to the polycrystallinity and/or the inhomogeneity of the layers, a homogeneous diffusion of molecules into the MOF layer is impossible or at least highly limited. Also, in membrane applications, inhomogeneous films of strongly varying thickness containing a number of pin-holes are not very attractive, since these defects will severely limit separation efficiency.

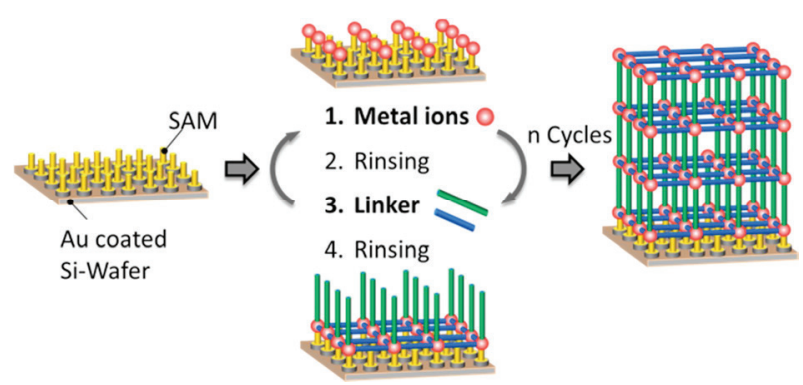

Fig. 1. Scheme of the layer-by-layer liquid phase epitaxy process for SURMOF preparation. Depending on the substrate material for SURMOF growth, the surface has to be activated either by plasma treatment or by the formation of a self-assembled monolayer (SAM). The horizontal and vertical linker molecules do not necessarily have to be different. 
To overcome these limitations, a method was developed in 2007 based on a layer-by-layer (LBL) liquid phase epitaxy (LPE) that produces very smooth, homogeneous and highly oriented surface-anchored metal-organic framework (SURMOF) coatings with uniform layer thickness [6]. For SURMOF preparation, meanwhile several deposition methods have been developed, ranging from robot supported dipping methods to automated spray processes $[7,8]$.

Once a SURMOF is grown it can be used as a host structure for guest molecules. Loading of a SURMOF with the guest molecules will change the effective refractive index of the surface film. This change in optical properties can be detected by surface sensitive optical sensors.

In this paper we will demonstrate the high potential of the SURMOFs as a new kind of platform material for optical sensor devices based on a change in the refractive index. While recent papers focus on classical detection methods such as ellipsometry [9], here we use nanoplasmonic sensing (NPS), based on localized surface plasmon resonance (LSPR) as optical detection method. In NPS, a glass substrate is coated with a close-range ordered array of gold nanodisks and covered by a protective layer of $\mathrm{SiO}_{2}$ (Figure 2). The plasmon resonance wavelength of the gold nanodisks on the NPS sensor will depend on the optical properties of the immediately (few tens of nanometers) adjacent media [10]. By coating the sensor chip with porous SURMOFs - here we used HKUST-1 [11] - and upon loading the SURMOF pores with guest molecules, the effective refractive index of the SURMOF layer changes, resulting in a proportional shift of the LSPR wavelength (Figure 3).

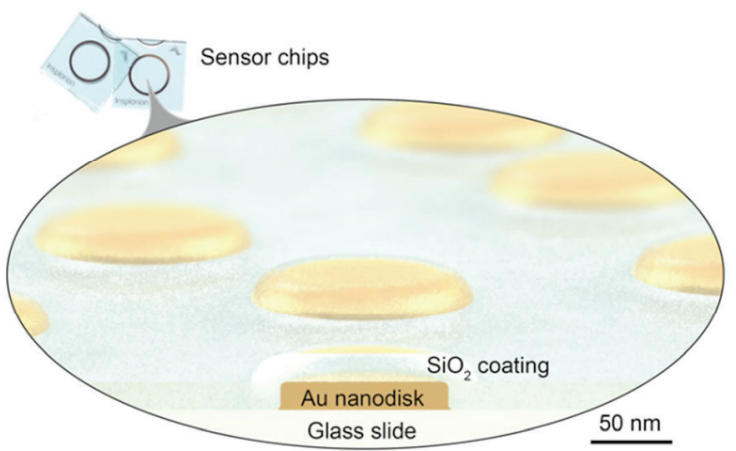

Fig. 2. Schematic setup of a typical sensor chip used for nanoplasmonic sensing.
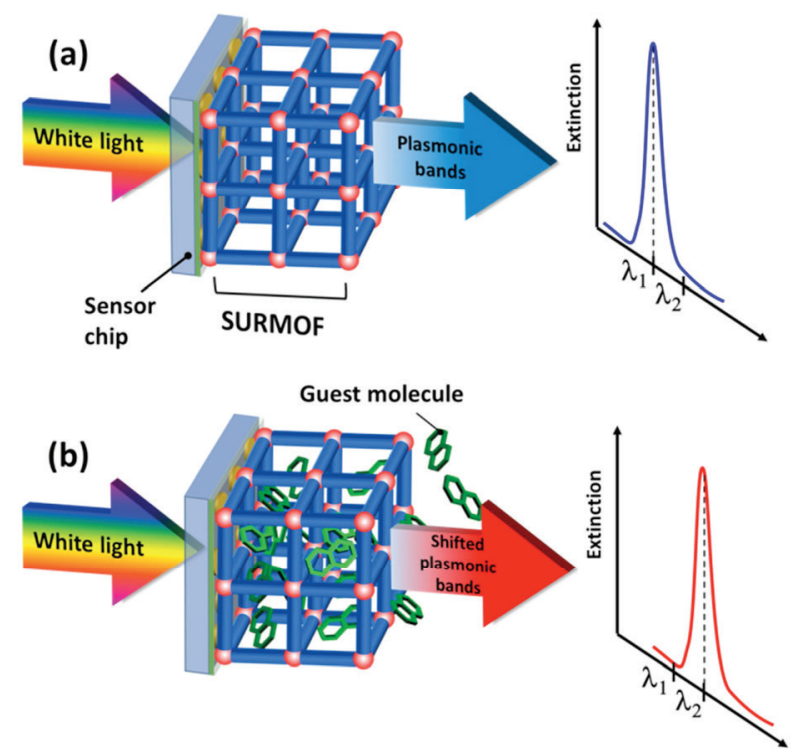

Fig. 3. When white light passes through the SURMOF coated sensor chip the plasmonic band of the gold nanodisks can be detected in transmission (a). After loading the SURMOF with guest molecules, the effective refractive index around the gold nanodisks changes, resulting in a spectral shift of the plasmonic band (b).

\section{Materials and Methods}

NPS sensor chips, coated with $\mathrm{SiO}_{2}$ (Insplorion $A B$, Sweden) were used as substrates for SURMOF deposition. In a first step the substrates were treated with oxygen plasma to clean the $\mathrm{SiO}_{2}$ surface and to functionalize it with $-\mathrm{OH}$ groups, which support the SURMOF growth. After this activation procedure a layer-by-layer spray deposition of the SURMOF HKUST-1 was carried out by spraying different liquids in the following sequence onto the substrate: (1) a $1 \mathrm{mM}$ ethanolic copper acetate solution (= metal source), (2) pure ethanol for rinsing, (3) a $0.2 \mathrm{mM} \mathrm{1,3,5}$ benzene tricarboxylic acid (BTC) solution (= linker source) and (4) again pure ethanol for rinsing. This deposition procedure was repeated 5,15 and 20 times resulting in a SURMOF thickness of about 40, 120 and $180 \mathrm{~nm}$ respectively. For the nanoplasmonic sensing experiment the experimental setup shown in Figure 4 has been used. Argon was used as carrier gas for the guest molecules (cyclohexane or ethanol). To load the SURMOFs with guest molecules the argon was bubbled through a washing bottle filled with cyclohexane or ethanol and afterwards guided to the measuring chamber. To drive a base line and to remove the guest molecules from the pores, the valves were switched in such way that the pure argon was directed to the measuring cell. For the 
duration of the experiment extinction spectra were recorded at room temperature and the position of the plasmon resonance wavelength was detected using the XNano equipment (Insplorion, Sweden) at a time resolution of $1 \mathrm{~Hz}$.

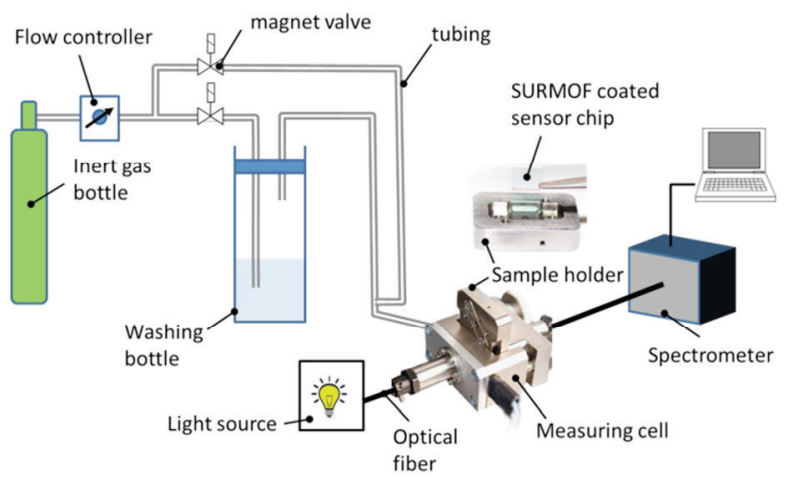

Fig. 4. Schematic setup of the nanoplasmonic sensing experiment.

\section{Results and Discussion}

For a first experiment the sensor chips were coated with 5 and 15 layers of HKUST-1 SURMOF and cyclohexane was used as probe molecule. A sensor chip without SURMOF was used as reference sample. In Figure 5 the spectral shift of the plasmon resonance wavelength is shown as a function of time and SURMOF layer thickness. Before the argon was bubbled through the cyclohexane to load the SURMOF pores, for all samples a base line was recorded by flooding the measuring cell with pure carrier gas. In the graph the base lines were all set to "zero". After 2 minutes (orange arrow in Figure 5) the valves were switched and the argon was bubbled through the cyclohexane. For all three samples a red shift in the spectra can be observed, which is significantly pronounced in the case of SURMOF coated samples. This observation can be explained with the probe depth of the NPS technique, which can detect changes in the refractive index in a layer of up to $30 \mathrm{~nm}$ thickness above the gold nanodisks. In the case of the reference sample the refractive index around the gold nanodisks changes (1) by the cyclohexane in the gas phase and (2) by the decoration of the sensor chip surface with adsorbed cyclohexane molecules, which leads to the formation of a molecular layer, where the density of cyclohexane molecules is increased compared to that in the gas phase but which is much thinner than the probe depth of the method.

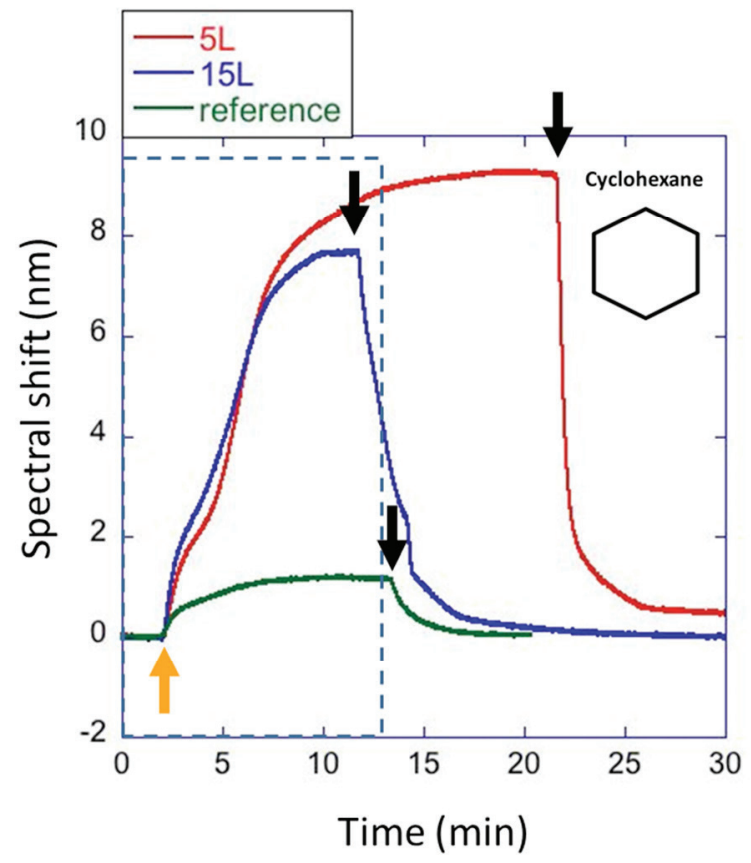

Fig. 5. Spectral shift of the plasmonic band of the gold nanodisks as a function of time and HKUST-1 layer thickness after cyclohexane was supported (orange arrow). The black arrows mark the switch from cyclohexane/argon mixture to pure argon.

In the case of SURMOF coated samples, however, we find a different situation: Due to the adsorption of the cyclohexane molecules inside the SURMOF pores the density of the probe molecules compared to those in the gas phase increases and with that the refractive index around the gold nanodisks. However, in contrast to the reference sample, here the layer with increased cyclohexane density is much thicker and can be even thicker than the probe depth of the NPS (see Figure 6). As soon as the support with cyclohexane is switched off (black arrows in Figure 5) pure argon flows through the measuring chamber and the cyclohexane molecules in the pores diffuse out, resulting in the decrease of the refractive index and a corresponding blue shift of the plasmonic band. 


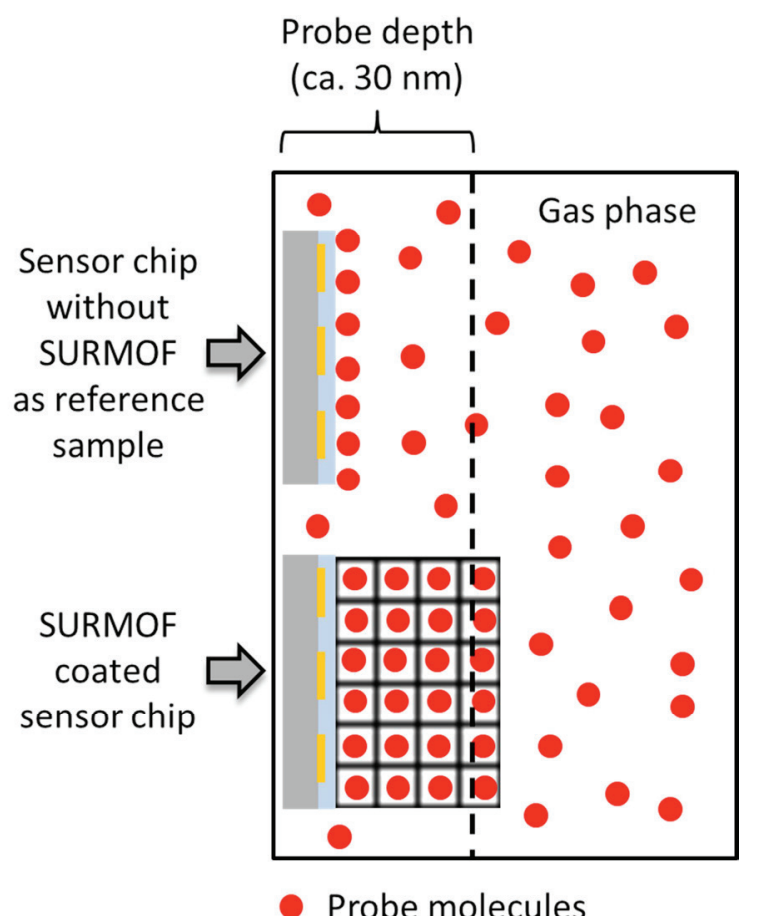

Fig. 6. Scheme for the explanation of the increase of probe molecule density in the vicinity of the gold nanodisks by a SURMOF coating.

The final nanoplasmonic shifts for the 5 layer $(5 \mathrm{~L})$ and the 15 layer $(15 \mathrm{~L})$ sample after ethanol loading are comparable, which might be explained by a SURMOF layer thickness which is in both cases higher than the probe depth of the method.

Figure 7 shows the results of NPS experiment with a 20 layer HKUST-1 coated sample and ethanol as probe molecule. The orange arrows mark the switch from pure argon to the ethanol/argon mixture, while the black arrows mark the switch back to pure argon. In both cycles the spectral position of the plasmonic band reaches the original base line level after purging with pure argon, demonstrating that the ethanol molecules diffuse out of the pores completely. Comparing the kinetics of the loading of the HKUST-1 structures with cyclohexane (Figure 5) and with ethanol (Figure 7) the spectral shift in the latter case occurs faster. This observation could be explained with the bimodal pore size distribution of the HKUST-1 SURMOF consisting of small hydrophobic pores (diameter $0.6 \mathrm{~nm}$ ) and bigger hydrophilic pores (diameter $0.9 \mathrm{~nm}$ ) [12]. Accordingly the bigger pores - and thus most of the volume of the SURMOF - will be preferentially filled with the polar ethanol molecules, while the nonpolar cyclohexane will primarily occupy the smaller pores. As a result more ethanol molecules will be loaded in the big SURMOF pores per time unit than cyclohexane molecules in the smaller pores.

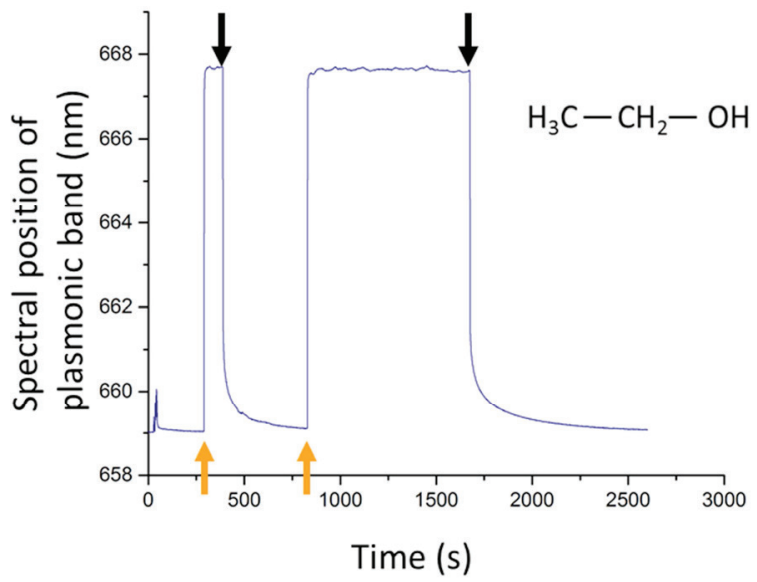

Fig. 7. Spectral position of the plasmonic band of the gold nanodisks as a function of time for a sensor chip coated with HKUST-1 and treated with ethanol/argon. The orange arrows mark the switch from pure argon to the argon/ethanol mixture, the black arrows the switch back to pure argon.

\section{Conclusion}

It was demonstrated that highly porous surfaceanchored metal-organic frameworks can change the effective refractive index by the adsorption of guest molecules, which can be detected indirectly by the method of nanoplasmonic sensing. As SURMOFs and SURMOF-based materials can be produced in a well-defined manner with respect to pore geometries and properties, this material class is predestinated as platform for the development of gas sensors specifically sensitive for a certain gas or gas class.

\section{Acknowledgements}

$\mathrm{H}$. Gliemann thanks the KIT for funding related to the TT-project N032 and the Baden-Württemberg Stiftung for financial support.

\section{References}

[1] D. Zacher, A. Baunemann, S. Hermes, R. A. Fischer, Deposition of microcrystalline $\left[\mathrm{Cu}_{3}(\mathrm{btc})_{2}\right]$ and $\left[\mathrm{Zn}_{2}(\mathrm{bdc})_{2}(\right.$ dabco $\left.)\right]$ at alumina and silica surfaces modified with patterned self assembled organic monolayers: evidence of surface selective and oriented growth, Journal of Materials Chemistry 17, 2785-2792 (2007); DOI: 10.1039/b703098c 
[2] G. Lu, J. T. Hupp, Metal-Organic Frameworks as Sensors: A ZIF-8 Based Fabry-Perot Device as a Selective Sensor for Chemical Vapors and Gases, Journal of the American Chemical Society 132, 7832-7833 (2010); DOI: 10.1021/ja101415b

[3] E. Biemmi, C. Scherb, T. Bein, Oriented growth of the metal organic framework $\mathrm{Cu}_{3}(\mathrm{BTC})_{2}\left(\mathrm{H}_{2} \mathrm{O}\right)_{3} \quad \mathrm{xH}_{2} \mathrm{O}$ tunable with functionalized self-assembled monolayers, Journal of the American Chemical Society 129, 8054-8055 (2007); DOI: 10.1021/ja0701208

[4] M. Tsotsalas, A. Umemura, F. Kim, Y. Sakata, J. Reboul, S. Kitagawa, S. Furukawa, Crystal morphology-directed framework orientation in porous coordination polymer films and freestanding membranes via LangmuirBlodgettry, Journal of Materials Chemistry 22, 10159-10165 (2012); DOI:

10.1039/c2jm16333k

[5] P. Falcaro, R. Ricco, C. M. Doherty, K. Liang, A. J. Hill, M. J. Styles, MOF positioning technology and device fabrication, Chemical Society Reviews 43, 5513-5560 (2014); DOI: $10.1039 / \mathrm{c} 4 \mathrm{cs} 00089 \mathrm{~g}$

[6] O. Shekhah, H. Wang, S. Kowarik, F. Schreiber, M. Paulus, M. Tolan, C. Sternemann, F. Evers, D. Zacher, R. A. Fischer, C. Woll, Step-by-step route for the synthesis of metal-organic frameworks, Journal of the American Chemical Society 129, 1511815119 (2007); DOI: 10.1021/ja076210u

H. K. Arslan, O. Shekhah, J. Wohlgemuth, M. Franzreb, R. A. Fischer, C. Woll, HighThroughput Fabrication of Uniform and Homogenous MOF Coatings, Advanced Functional Materials 21, 4228-4231 (2011); DOI: 10.1002/adfm.201101592

[8] Z.-G. Gu, A. Pfriem, S. Hamsch, H. Breitwieser, J. Wohlgemuth, L. Heinke, H. Gliemann, C. Woell, Transparent films of metal-organic frameworks for optical applications, Microporous and Mesoporous Materials 211, 82-87 (2015); DOI:

10.1016/j.micromeso.2015.02.048

[9] E. Redel, Z. Wang, S. Walheim, J. Liu, H. Gliemann, C. Woell, On the dielectric and optical properties of surface-anchored metalorganic frameworks: A study on epitaxially grown thin films, Applied Physics Letters 103, (2013); DOI: 091903 10.1063/1.4819836

[10] E. M. Larsson, C. Langhammer, I. Zorić, B. Kasemo, Nanoplasmonic Probes of Catalytic Reactions, Science 326, 1091-1094 (2009); DOI: 10.1126/science.1176593

[11] S. S. Y. Chui, S. M. F. Lo, J. P. H. Charmant, A. G. Orpen, I. D. Williams, A chemically functionalizable nanoporous material $\left[\mathrm{Cu}_{3}(\mathrm{TMA})_{2}\left(\mathrm{H}_{2} \mathrm{O}\right)_{3}\right]_{\mathrm{n}}$, Science $283,1148-1150$ (1999); DOI: 10.1126/science.283.5405.1148

[12] P. Kusgens, M. Rose, I. Senkovska, H. Frode, A. Henschel, S. Siegle, S. Kaskel, Characterization of metal-organic frameworks by water adsorption, Microporous and Mesoporous Materials 120, 325-330 (2009); DOI: 10.1016/j.micromeso.2008.11.020 